\title{
A Stretched Icosahedral Grid by a New Grid Transformation
}

\author{
Hirofumi TOMITA \\ Frontier Research Center for Global Change, JAMSTEC, Yokohama, Japan \\ (Manuscript received 28 August 2007, in final form 7 April 2008)
}

\begin{abstract}
This study developed a general grid transformation method for a horizontal grid system on a sphere. The method incorporates the currently used Schmidt transformation method, as well as a new technique that includes intuitive interpretation of the Schmidt transformation. To apply the method, we developed an estimation function that considers both isotropy and homogeneity, and the transformation function uses a governing differential equation to ensure that the function takes the minimum value. Since the proposed transformation method avoids the too-fine grid at the center of the target region, which arises due to the Schmidt transformation method, the new method is superior in terms of computational efficiency.

We applied the new transformation to an icosahedral grid. To investigate the stretching effect by this method, we conducted an advection test case using the standard experiment for the shallow water model (Williamson et al. 1992). The error growth rate was minimized over the target region where the fine grid area was distributed. The transition zone between the target region and the coarser grid region exhibited smooth advection, so no spurious error occurred.
\end{abstract}

\section{Introduction}

For reasons related to computational and physical performance, researchers have recently revived the use of grid-point methods on polyhedral grids over a sphere in atmospheric and oceanic general circulation modeling. For example, the Conformal Cubic Atmospheric Model (CCAM) of the Commonwealth Scientific and Industrial Research Organisation (CSIRO) is based on a cubic grid (McGregor and Dix 1997, 1998). An icosahedral grid is another promising choice. The first applications of the icosahedral grid to a geophysical problem were published by Williamson (1968) and Sadourny et al. (1968). Since these were published, many researchers have attempted to develop models using the icosahedral grid (Baumgardner and Frederickson 1985; Cullen and Hall 1979; Masuda

Corresponding author: Hirofumi Tomita, Frontier Research Center for Global Change, JAMSTEC, 3173-25, Showa-machi, Kanazawa-ku, Yokohama, Kanagawa 236-0001, Japan

E-mail: htomita@jamstec.go.jp

(C)2008, Meteorological Society of Japan and Ohnishi 1986; Stuhne and Peltier 1996). Successful examples include the Colorado State University (CSU) atmospheric general circulation model (AGCM) (Heikes and Randall 1995a, b; Ringler et al. 2000) and the GME prediction model of the Deutscher Wetterdienst (German Weather Service) (Majewski et al. 2002).

Our research group has been developing Nonhydrostatic Icosahedral Atmospheric Model (NICAM). This AGCM is a new type of global atmospheric model that employs a new nonhydrostatic scheme (Satoh 2002, 2003) and a modified icosahedral grid (Tomita et al. 2002, 2001). For a detailed description of the dynamical core and recent status of NICAM, see Tomita and Satoh (2004) and Satoh et al. (2008), respectively. Use of the global NICAM cloud-resolving model has led to several accomplishments, including the first global cloud-resolving simulation on the aqua planet (Tomita et al. 2005), a climate sensitivity test by a global cloud-resolving model (Miura et al. 2005), a simulation of multi-scale cloud organization (Nasuno et al. 2007), a short-term simulation based on realistic topography (Miura et al. 2007b), 
a resolution-dependency test for the dynamical core (Iga et al. 2007), and a Madden-Julian Oscillation simulation (Miura et al. 2007a). Thus, the icosahedral grid has proven to be very useful for super high-resolution simulations.

However, when examining a local area with a finer structure, regional models may be more appropriate than global models. Because regional models normally use boundary conditions generated from global models, this can complicate boundary treatment and may worsen solution precision due to the artificial reflection of waves at the boundary. Straightforward use of a quasi-uniform grid system for regional climate or weather studies is inefficient and requires very high computational cost, but techniques for stretching horizontal grids can resolve these problems. Advantages of this technique include the avoidance of abrupt wave reflections and utilization of the same dynamical core in both the local area and global domain.

McGregor (1997b) applied the Schmidt transformation (Schmidt 1977) to a cubic grid model and successfully simulated local fine structures (McGregor 1997a, 1999; McGregor and Katzfey 1998, 1999). One drawback of the Schmidt transformation is nonuniform resolution in a local region of interest, with resolution lessening with increasing distance from the central point of the target region (see Fig. 3 in Courtier and Geleyn 1988). The Courant-Friedrichs-Lewy (CFL) condition restricts the available time interval, which is determined by the minimum grid size at the central point. To maximize computational efficiency, having a relatively homogeneous grid distribution in the target region is desirable. The purpose of this study was to ameliorate this problem.

The next section of this paper discusses preferable features of a transformation function to resolve a local area using a stretched grid. Section 3 introduces a new transformation function that takes into account the drawbacks of the Schmidt transformation. Section 4 presents an actual application of the new transformation and compares its properties to results using the Schmidt transformation. It also presents the advection test case set out by Williamson et al. (1992) on the icosahedral grid stretched by the proposed transformation, confirming the validity of the new transformation. Finally, Section 5 presents our concluding remarks.

\section{Grid transformation on a sphere}

\subsection{Preferable properties of grid transformation}

A transformation function of a uniform grid system has certain preferable conditions. In particular, we focused on a local region and tried to resolve that region to a finer mesh. Hereafter, we refer to the local region of interest as the target region.

Condition (a): Isotropy in the target region

The shape of a grid element (control volume in the case of the finite volume method) should not vary greatly after transformation. Here, this property is termed isotropy. This condition may seem identical to conformality, but the two actually differ. For example, the transformation $h(z)$ $=1 / z$ in a complex plane, where $z$ is complex, is a conformal mapping but is not an isotropic mapping; a line triangle element is transformed to a curved triangle with angle conservation but with shape distortion.

Condition (b): Homogeneity in the target region The grid interval in the target region should be as constant as possible. This property is termed homogeneity. The ratio of the maximum grid interval to the minimum grid interval affects computational efficiency.

Condition (c): Smoothness of the transformation functions

The transformation must be continuous and should be smooth; that is, the transformation function should be differentiable because the derivative of the transformation function against the original latitude represents the grid interval at that latitude. Otherwise, if the grid interval abruptly changes, wave reflection could occur in that region.

Condition (d): A few parameters for the construction of the transformation

The transformation functions should be constructed using only a few parameters. If several parameters are involved, identifying the best transformation function becomes difficult. In addition, the meaning of the parameters should be clear.

\subsection{Isotropic transformation}

First, we construct a transformation function to satisfy the isotropy condition (condition (a)). Figure 1 schematizes the grid transformation, with the target region around the South Pole. The radius of the sphere is one. Let the number of grid points be 


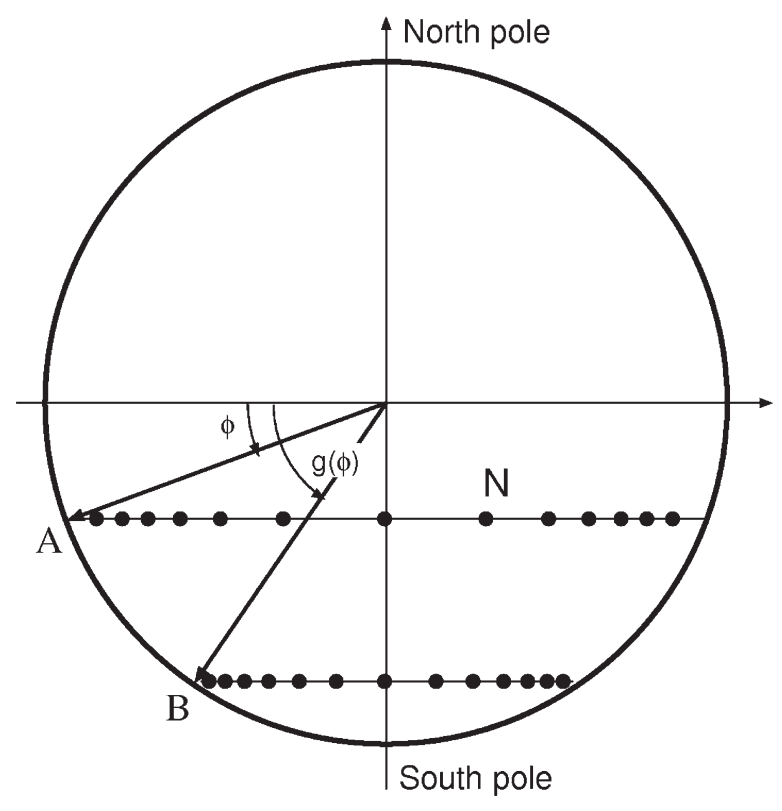

Fig. 1. Schematic figure of isotropic grid transformation from a quasi-uniform grid.

$N$ at latitude A, the original latitude be $\phi$, the transformed latitude be $\varphi$, and the transformation function be $g$. Since the length of the latitudinal circle is given by $2 \pi \cos \phi$, the mean longitudinal grid interval at latitude $\mathrm{A}$, denoted as $\Delta x$, is given by

$$
\Delta x=\frac{2 \pi \cos \phi}{N} .
$$

In the quasi-uniform grid, the mean grid interval in the latitudinal direction, denoted as $\Delta y$, is almost equal to that in the longitudinal direction:

$$
\Delta y \simeq \Delta x .
$$

Since the unit sphere is assumed here, the grid interval in the latitudinal direction is the same as the interval of latitude:

$$
\Delta y \simeq \Delta \phi .
$$

By transformation $g(\phi)$, let latitude A be transformed to latitude B. Since the length of the latitudinal circle at latitude B is given by $2 \pi \cos [g(\phi)]$, the mean longitudinal grid interval $\Delta x^{\prime}$ at latitude $B$ is given by

$$
\Delta x^{\prime}=\frac{2 \pi \cos [g(\phi)]}{N} .
$$

By the isotropic constraint, the grid interval $\Delta y^{\prime}$ in the latitudinal direction is the same as that in the longitudinal direction:

$$
\Delta y^{\prime} \simeq \Delta x^{\prime} .
$$

In the same way as Eq. (3), the grid interval in the latitudinal direction is the same as the interval of latitude due to the assumption of the unit sphere:

$$
\Delta y^{\prime} \simeq \Delta \varphi .
$$

From Eq. (1)-Eq. (6), we can easily derive the following ordinary differential equation within the limit $\Delta \phi \rightarrow 0$ :

$$
\frac{d g(\phi)}{d \phi}=\frac{\cos [g(\phi)]}{\cos \phi} .
$$

This equation is identical to that in the Schmidt transformation, so this isotropic transformation is equivalent to the Schmidt transformation. The Schmidt transformation was originally introduced as a conformal mapping technique, so the derivation of the differential equation of Eq. (7) from Eq. (1)-Eq. (6) is another interpretation.

\subsection{Homogeneous transformation}

While the Schmidt transformation yields perfect isotropy, it sacrifices homogeneity (condition (b)). Let us consider transformation of a quasi-homogeneous grid, maintaining a constant latitudinal grid interval within the target region:

$$
\frac{d g(\phi)}{d \phi}=\beta,
$$

where $\beta$ is a constant value. In this case, the longitudinal grid interval becomes longer than the latitudinal grid interval: $\Delta x^{\prime}>\Delta y^{\prime}$. Thus, homogeneity is incompatible with isotropy. Note that no completely homogeneous transformation exists for two orthogonal directions on a sphere.

\section{A new grid transformation}

\subsection{Formulation}

As noted in the previous section, no transformation function can yield both perfect isotropy and homogeneity. This section introduces our proposed grid transformation technique, which is based on the relative importance of isotropy and homogeneity. Let the latitude of the grid location $\phi$ again be transformed to $\varphi$ by the function $g(\phi)$. Isotropy and latitudinal homogeneity at the new latitude $\varphi$ can be estimated by 


$$
\begin{aligned}
& e_{i}=\left.\left(\frac{d g(\phi)}{d \phi}-\frac{\cos [g(\phi)]}{\cos \phi}\right)^{2}\right|_{\varphi=g(\phi)}, \\
& e_{h}=\left.\left(\frac{d g(\phi)}{d \phi}-\beta\right)^{2}\right|_{\varphi=g(\phi)},
\end{aligned}
$$

respectively ${ }^{1}$.

When Eqs. (9) and (10) are multiplied by the appropriate weighting functions, combined, and then integrated from latitude $a^{\prime}$ to latitude $b^{\prime}$, a gross measure for both the homogeneity and the isotropy can be derived as

$$
I=2 \pi \int_{a^{\prime}}^{b^{\prime}}\left[\tilde{A}(\varphi)\left(\dot{\varphi}-\frac{\cos \varphi}{\cos \phi}\right)^{2}+\tilde{B}(\varphi)(\dot{\varphi}-\beta)^{2}\right] \cos \varphi d \varphi,
$$

where $\dot{\varphi}=d \varphi / d \phi, \tilde{A}(\varphi)$ and $\tilde{B}(\varphi)$ are weighting functions. Since $d \varphi=\dot{\varphi} d \phi$, the integration $I$ can be written as a function of $\phi$ :

$$
I(\phi)=\int_{a}^{b}\left[A(\varphi)\left(\dot{\varphi}-\frac{\cos \varphi}{\cos \phi}\right)^{2}+B(\varphi)(\dot{\varphi}-\beta)^{2}\right] \dot{\varphi} d \phi,
$$

where

$$
\begin{aligned}
& A(\varphi) \equiv 2 \pi \tilde{A}(\varphi) \cos \varphi, \quad B(\varphi) \equiv 2 \pi \tilde{B}(\varphi) \cos \varphi, \\
& a=g^{-1}\left(a^{\prime}\right), \quad b=g^{-1}\left(b^{\prime}\right) .
\end{aligned}
$$

If we define function $F(\phi, \varphi, \dot{\varphi})$ as

$$
F(\phi, \varphi, \dot{\varphi})=\left[A(\varphi)\left(\dot{\varphi}-\frac{\cos \varphi}{\cos \phi}\right)^{2}+B(\varphi)(\dot{\varphi}-\beta)^{2}\right] \dot{\varphi},
$$

the requirement that measure $I$ should be minimal leads to the Euler equation:

$$
\frac{\partial F}{\partial \varphi}-\frac{d}{d \phi}\left[\frac{\partial F}{\partial \dot{\varphi}}\right]=0
$$

in which functions $A$ and $B$ are externally specified. The following relationships are obtained:

1 Although $d^{2} g(\phi) / d \phi^{2}$ can be used to estimate homogeneity instead of Eq. (10), this results in a higherorder differential equation in the subsequent derivation of the Euler equation. This type of equation is difficult to solve, and estimates of $d^{2} g(\phi) / d \phi^{2}$ do not contain information about the degree of homogeneity $\beta$. Therefore, we used Eq. (10) to estimate homogeneity.

$$
\begin{aligned}
& \frac{\partial F}{\partial \varphi}=B^{\prime}(\varphi) \dot{\varphi}(\dot{\varphi}-\beta)^{2}+A^{\prime}(\varphi) \dot{\varphi}\left(\dot{\varphi}-\frac{\cos \varphi}{\cos \phi}\right)^{2} \\
& +2 A(\varphi) \dot{\varphi} \frac{\sin \varphi}{\cos \phi}\left(\dot{\varphi}-\frac{\cos \varphi}{\cos \phi}\right), \\
& \frac{\partial F}{\partial \dot{\varphi}}=B(\varphi)(\dot{\varphi}-\beta)^{2}+2 B(\varphi) \dot{\varphi}(\dot{\varphi}-\beta) \\
& +A(\varphi)\left(\dot{\varphi}-\frac{\cos \varphi}{\cos \phi}\right)^{2}+2 A(\varphi) \dot{\varphi}\left(\dot{\varphi}-\frac{\cos \varphi}{\cos \phi}\right), \\
& \frac{d}{d \phi}\left[\frac{\partial F}{\partial \dot{\varphi}}\right]=2 B(\varphi) \dot{\varphi} \ddot{\varphi}+4 B(\varphi)(\dot{\varphi}-\beta) \ddot{\varphi} \\
& +B^{\prime}(\varphi) \dot{\varphi}(\dot{\varphi}-\beta)+2 B^{\prime}(\varphi) \dot{\varphi}^{2}(\dot{\varphi}-\beta) \\
& +2 A(\varphi)\left(\dot{\varphi}-\frac{\cos \varphi}{\cos \phi}\right) \ddot{\varphi} \\
& +2 A(\varphi) \dot{\varphi}\left(\ddot{\varphi}-\frac{\sin \phi \cos \varphi}{\cos { }^{2} \phi}+\frac{\dot{\varphi} \sin \phi}{\cos \phi}\right) \\
& +2 A(\varphi)\left(\dot{\varphi}-\frac{\cos \varphi}{\cos \phi}\right)\left(\ddot{\varphi}-\frac{\sin \phi \cos \varphi}{\cos { }^{2} \phi}+\frac{\dot{\varphi} \sin \phi}{\cos \phi}\right) \\
& +A^{\prime}(\varphi) \dot{\varphi}\left(\dot{\varphi}-\frac{\cos \varphi}{\cos \phi}\right)^{2}+2 A^{\prime}(\varphi) \dot{\varphi}^{2}\left(\dot{\varphi}-\frac{\cos \varphi}{\cos \phi}\right)
\end{aligned}
$$

In addition, the natural boundary condition must fulfill the relationship $\partial F / \partial \dot{\varphi}=0$ at $\phi=a, b$ :

$$
\begin{aligned}
& B(\varphi)(\dot{\varphi}-\beta)^{2}+2 B(\varphi) \dot{\varphi}(\dot{\varphi}-\beta) \\
& +A(\varphi)\left(\dot{\varphi}-\frac{\cos \varphi}{\cos \phi}\right)^{2}+\left.2 A(\varphi) \dot{\varphi}\left(\dot{\varphi}-\frac{\cos \varphi}{\cos \phi}\right)\right|_{\dot{\phi}=a, b}=0 .
\end{aligned}
$$

Thus, the problem is replaced by the solution of the second-order ordinary differential Eq. (13) constructed using Eqs. (14)-(16) with the two boundary conditions (17).

When the transformation function covers the sphere,

$$
\begin{aligned}
& \lim _{a \rightarrow-\pi / 2+0} a^{\prime}=-\frac{\pi}{2}, \\
& \lim _{b \rightarrow \pi / 2-0} b^{\prime}=\frac{\pi}{2} .
\end{aligned}
$$

The weighting functions $A(\varphi)$ and $B(\varphi)$ should be consistent with Eq. (17) and Eqs. (18)-(19). Furthermore, to satisfy condition (c), $A(\varphi)$ and $B(\varphi)$ must be differentiable. 


\subsection{Method to obtain solutions}

Since Eq. (13) is nonlinear with Eqs. (14)-(16), obtaining analytical solutions is difficult. We can numerically solve the equations using the following method. Introducing variables $\varphi_{0}=\varphi$ and $\varphi_{1}=$ $\dot{\varphi}$, we can rewrite the equations as a set of firstorder ordinary equations:

$$
\begin{aligned}
& \dot{\varphi}_{0}=f_{0}\left(\phi, \varphi_{0}, \varphi_{1}\right), \\
& \dot{\varphi}_{1}=f_{1}\left(\phi, \varphi_{0}, \varphi_{1}\right),
\end{aligned}
$$

where

$$
\begin{aligned}
& f_{0}\left(\phi, \varphi_{0}, \varphi_{1}\right)=\varphi_{1},(22) \\
& f_{1}\left(\phi, \varphi_{0}, \varphi_{1}\right) \\
& =\left[-A^{\prime}\left(\varphi_{0}\right) \varphi_{1}^{2}\left(\varphi_{1}-\frac{\cos \varphi_{0}}{\cos \phi}\right)-B^{\prime}\left(\varphi_{0}\right) \varphi_{1}^{2}\left(\varphi_{1}-\beta\right)\right. \\
& \quad-A\left(\varphi_{0}\right) \varphi_{1}\left(\frac{\varphi_{1} \sin \varphi_{0}}{\cos \phi}-\frac{\sin \phi \cos \varphi_{0}}{\cos ^{2} \phi}\right) \\
& \quad-A\left(\varphi_{0}\right)\left(\varphi_{1}-\frac{\cos \varphi_{0}}{\cos \phi}\right)\left(\frac{\varphi_{1} \sin \varphi_{0}}{\cos \phi}-\frac{\sin \phi \cos \varphi_{0}}{\cos ^{2} \phi}\right) \\
& \left.+A\left(\varphi_{0}\right) \varphi_{1} \frac{\sin \varphi_{0}}{\cos \phi}\left(\varphi_{1}-\frac{\cos \varphi_{0}}{\cos \phi}\right)\right] \\
& /\left[A\left(\varphi_{0}\right) \varphi_{1}+B\left(\varphi_{0}\right) \varphi_{1}\right. \\
& \left.\quad+2 A\left(\varphi_{0}\right)\left(\varphi_{1}-\frac{\cos \varphi_{0}}{\cos \phi}\right)+2 B\left(\varphi_{0}\right)\left(\varphi_{1}-\beta\right)\right] .
\end{aligned}
$$

We can solve this system as an initial value problem instead of a boundary value problem (Eqs. 18 and 19).

Next, we examine the requirements for weighting functions $A$ and $B$. According to the conditions of Eq. (18),

$$
\lim _{\varphi_{0} \rightarrow-\pi / 2+0}\left(\varphi_{1}-\frac{\cos \varphi_{0}}{\cos \phi}\right)=0 .
$$

If $A(-\pi / 2)$ has a finite value, the following relation is necessary from Eq. (17):

$$
B\left(\varphi_{0}\right)\left(\varphi_{1}-\beta\right)^{2}+2 B\left(\varphi_{0}\right) \varphi_{1}\left(\varphi_{1}-\beta\right)=\left.0\right|_{\varphi_{0}=-\pi / 2} .
$$

We can specify the stretching ratio $\beta$ at the South Pole as $\varphi_{1}=\beta$. In this case, $B(-\pi / 2)$ can have any finite value.
Even if the initial values $\left(\varphi_{0}, \varphi_{1}\right)=(-\pi / 2, \beta)$ are given at $\phi=-\pi / 2$, fulfillment of Eq. (19) is not guaranteed. However, if $B(\pi / 2)=0$, Eq. (17) becomes

$$
\left.A\left(\varphi_{0}\right)\left(\varphi_{1}-\frac{\cos \varphi_{0}}{\cos \phi}\right)\left[1+2 \varphi_{1}\left(\varphi_{1}-\frac{\cos \varphi_{0}}{\cos \phi}\right)\right]\right|_{\varphi_{0}=\pi / 2}=0 .
$$

If and only if

$$
\lim _{\varphi_{0} \rightarrow \pi / 2-0}\left(\varphi_{1}-\frac{\cos \varphi_{0}}{\cos \phi}\right)=0,
$$

Equation (26) is satisfied even if $A(\pi / 2) \neq 0$, and Eq. (19) is simultaneously satisfied. Since $A(\varphi)$ is the isotropic weight (see Eq. 11), Eq. (27) should be fulfilled by increasing $A(\varphi)$ with $\varphi$. Thus, as an ultimate case, we can safely require

$$
\lim _{\varphi_{0} \rightarrow \pi / 2+0} A(\varphi)=\infty \text {. }
$$

Although $A(\varphi=-\pi / 2)$ is allowable as an arbitrary finite value to satisfy Eq. (25), it should be zero because homogeneity is more important at the central point of the target region. However, $B(\varphi=-\pi / 2)$ can be any nonzero positive value. In the outer region, where isotropy is more important, $B(\varphi)$ should be reduced to zero at $\varphi=\pi / 2$, and $A(\varphi)$ should become dominant.

\subsection{The Schmidt transformation as an initial value problem}

As described in the previous section, the Schmidt transformation is the solution to Eq. (7) with the two boundary conditions $\varphi= \pm \pi / 2$ at $\phi=$ $\pm \pi / 2$. By differentiating Eq. (7), we obtain

$$
\ddot{\varphi}=-\left(\frac{\dot{\varphi} \sin \varphi}{\cos \phi}-\frac{\sin \phi \cos \varphi}{\cos ^{2} \phi}\right) .
$$

Thus, the problem of Eq. (7) can be rewritten as a set of the first ordinary equations similar to Eqs. (20)-(23). In this case,

$$
f_{1}\left(\phi, \varphi_{0}, \varphi_{1}\right)=-\left(\frac{\varphi_{1} \sin \varphi_{0}}{\cos \phi}-\frac{\sin \phi \cos \varphi_{0}}{\cos ^{2} \phi}\right),
$$

with the initial condition $\left(\varphi_{0}=-\pi / 2, \varphi_{1}=\beta\right)$ at $\phi=$ $-\pi / 2$.

Based on the above discussion, the Schmidt transformation can be considered a special case of system equation (20)-(23) when $B(\varphi)=0$. In this 
case, Eq. (23) becomes

$$
\begin{aligned}
f_{1}\left(\phi, \varphi_{0}, \varphi_{1}\right) \\
=\left[-A^{\prime}\left(\varphi_{0}\right) \varphi_{1}^{2}\left(\varphi_{1}-\frac{\cos \varphi_{0}}{\cos \phi}\right)\right. \\
-A\left(\varphi_{0}\right) \varphi_{1}\left(\frac{\varphi_{1} \sin \varphi_{0}}{\cos \phi}-\frac{\sin \phi \cos \varphi_{0}}{\cos ^{2} \phi}\right) \\
-A\left(\varphi_{0}\right)\left(\varphi_{1}-\frac{\cos \varphi_{0}}{\cos \phi}\right)\left(\frac{\varphi_{1} \sin \varphi_{0}}{\cos \phi}-\frac{\sin \phi \cos \varphi_{0}}{\cos ^{2} \phi}\right) \\
\left.+A\left(\varphi_{0}\right) \varphi_{1} \frac{\sin \varphi_{0}}{\cos \phi}\left(\varphi_{1}-\frac{\cos \varphi_{0}}{\cos \phi}\right)\right] \\
/\left[A\left(\varphi_{0}\right) \varphi_{1}+2 A\left(\varphi_{0}\right)\left(\varphi_{1}-\frac{\cos \varphi_{0}}{\cos \phi}\right)\right] .
\end{aligned}
$$

If the following relation is fulfilled at $\phi=\phi_{i} \in[-\pi / 2$, $\pi / 2$ ]

$$
\varphi_{1}-\frac{\cos \varphi_{0}}{\cos \phi}=0,
$$

Equation (31) at $\phi=\phi_{i}$ becomes identical to Eq. (30); that is, $f_{1}\left(\phi, \varphi_{0}, \varphi_{1}\right)$ is independent of $A\left(\varphi_{0}\right)$. At $\phi=$ $\phi_{i}+\varepsilon$, where $\varepsilon$ is a positive infinitesimal, both Eqs. (32) and (30) are satisfied. Therefore, once Eq. (32) is fulfilled at $\phi=\phi_{i}$, the trajectory in the range $\varphi_{0}$ in $\phi>\phi_{i}$ is identical to the results using the Schmidt transformation.

\section{Transformation functions and the stretched grid}

The previous section described the formulation of the new transformation, the method of solution, and the constraint of weight functions $A(\varphi)$ and $B(\varphi)$. This section will present the actual determination of the weight functions based on their constraints and solve the equation system. This section will also compare the new transformation to the Schmidt transformation and discuss the properties of the transformation function. To validate the new transformation, we also performed an advection test case on the icosahedral grid stretched using the new transformation.

\subsection{Example of the transformation function}

Let the Southern Hemisphere be transformed into the target region, defined as the region $-\pi / 2$ $<\varphi<g(0)$. If we give a stretching parameter $\beta$, the Equator circle should be transformed around latitude $-\pi / 2(1-\beta)$. Therefore, we can roughly estimate the boundary of the target region $\varphi_{b}$ as

$$
\varphi_{b}=-\frac{\pi}{2}(1-\beta) \simeq g(0) .
$$

Note that $\varphi_{b}$ is not exactly the same as $g(0)$. We choose the following forms of the weighting functions $A(\varphi)$ and $B(\varphi): B(\varphi)$ is dominant in the target region $-\pi / 2<\varphi<\varphi_{b}$ so that homogeneity is weighted, while $A(\varphi)$ is dominant in the outer region $\varphi_{b}<$ $\varphi<\pi / 2$ so that isotropy is weighted:

$$
\begin{aligned}
& A(\varphi)= \begin{cases}\cos \left[\frac{\pi}{2}\left(\frac{\varphi-\varphi b}{-\pi / 2-\varphi b}\right)\right] & \varphi<\varphi_{b}, \\
1 / \cos ^{2}\left[\frac{\pi}{2}\left(\frac{\varphi-\varphi b}{\pi / 2-\varphi b}\right)\right] \quad & \varphi \geq \varphi_{b},\end{cases} \\
& A(\varphi)= \begin{cases}1 & \varphi \leq \varphi_{b}, \\
\frac{1}{2}\left(\cos \left[2 \pi \frac{(\varphi-\varphi b)}{(2 \varphi b+\pi)}\right]+1\right) & \varphi_{b}<\varphi \leq 2 \varphi_{b}+\pi / 2, \\
0 & \varphi>2 \varphi_{b}+\pi / 2 .\end{cases}
\end{aligned}
$$

In this case, only one parameter $\beta$ is given for $\mathrm{Eq}$. (33). Thus, condition (d) discussed in Section 2 is also satisfied. Figure 2 shows $A(\varphi)$ and $B(\varphi)$ at $\beta$ $=1 / 2$. These function forms satisfy the properties and constraints for $A(\varphi)$ and $B(\varphi)$, as discussed in the previous section.

The equation systems (20)-(23) under an initial condition $\left(\varphi_{0}, \varphi_{1}\right)=(-\pi / 2, \beta=1 / 2)$ at $\phi=-\pi / 2$ can be solved using the second-order Runge-Kutta method on the equidistant $\Delta \phi=\pi / n$, where $n$ is set to 5000 . The solid line in Fig. 3 indicates the generated transformation function. The Equator $(\phi$ $=0)$ is transformed to the latitudinal line $\varphi=g(0) \simeq$ -0.726 . Again, note that the lines of $\varphi_{b}$ and $g(0)$ differ slightly because the transformation does not have perfect homogeneity (see Fig. 2). The broken line in Fig. 3 represents the Schmidt transformation function for the case $g_{s}(0)=g(0)$, where $g_{s}$ indicates the Schmidt transformation function. Therefore, our proposed transformation yields the same number of grid points in the target region $-\pi / 2<\varphi<g(0)$ as the Schmidt transformation.

\subsection{Discussion for transformation functions}

As shown in Fig. 3, when we compare our results with those by the Schmidt transformation, we find that our system yields a more homogenous latitudinal resolution in the target region; the gradient is almost constant. In contrast, however, our transformation system expands the grid element in the longitudinal direction. Since the stretching fac- 

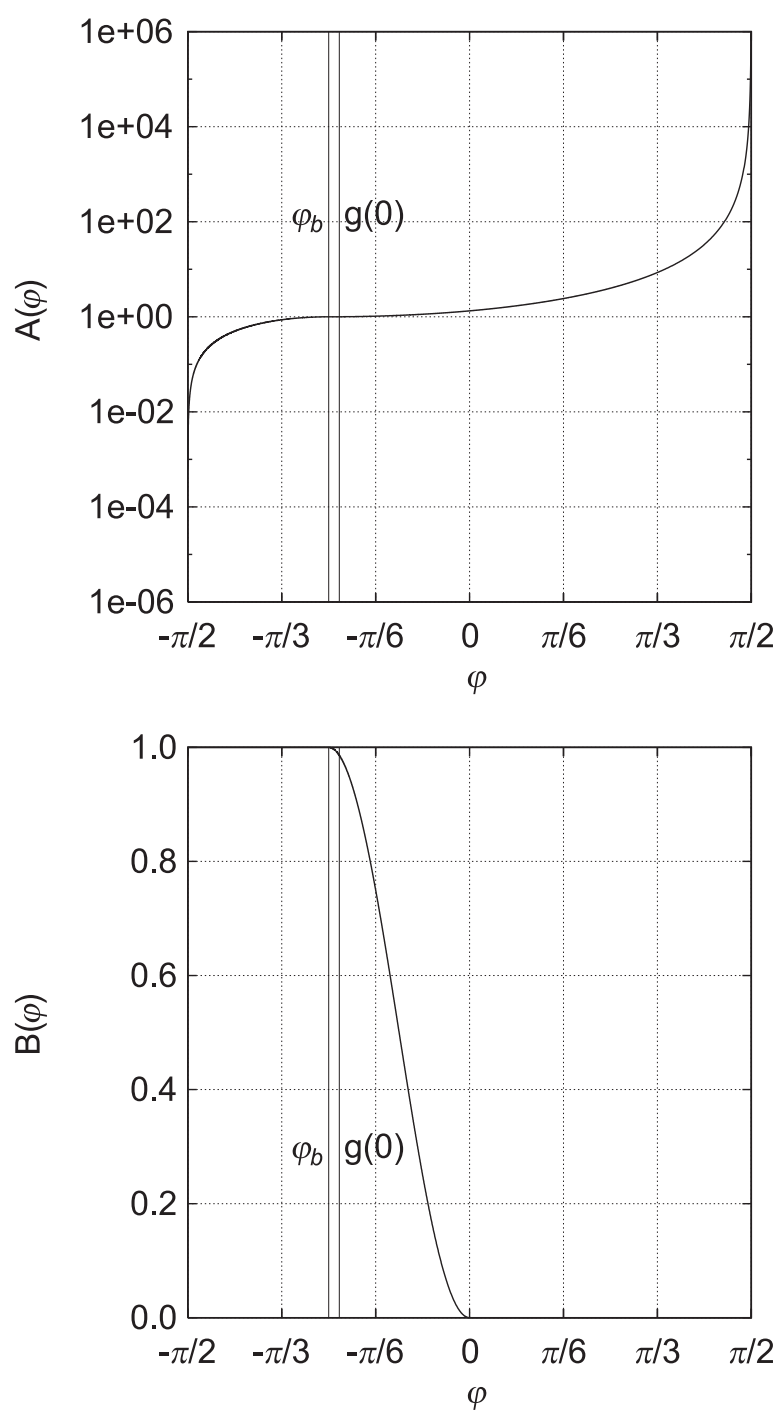

Fig. 2. The weighting functions $A$ and $B$ against the transformed latitude $\varphi(\beta=$ $1 / 2)$.

tor is estimated as $\cos \varphi \cos \phi$ in the longitudinal direction, we can define the aspect ratio of the longitudinal direction against the latitudinal one after a transformation as

$$
A R=\frac{\cos \varphi}{\cos \phi} / \dot{\varphi} .
$$

Note that in the Schmidt transformation, $A R$ is exactly unity everywhere. Figure 4 a shows the distribution of the aspect ratio against $\varphi$ for the present transformation. Although the aspect ratio gradually increases from unity with $\varphi$ in the target region,

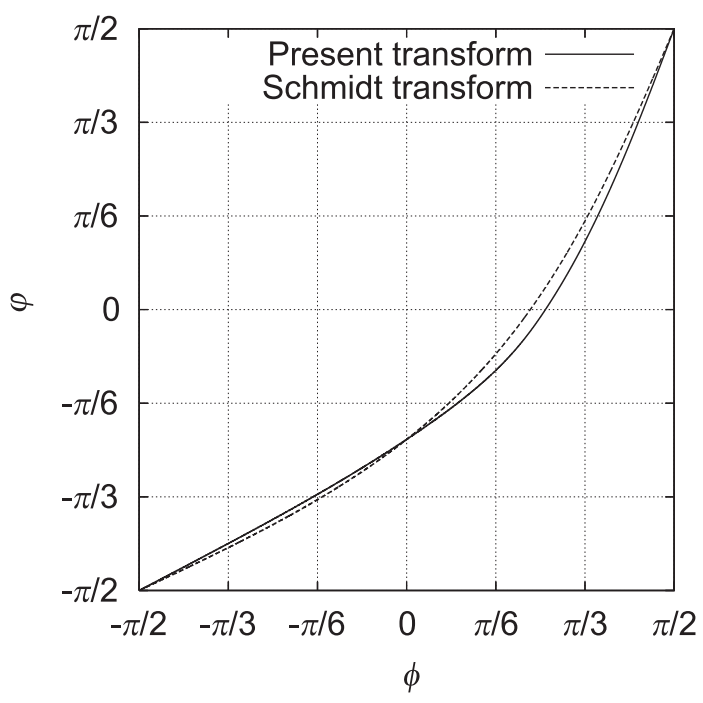

Fig. 3. The present transformation function with $\beta=1 / 2$ and the Schmidt transformation.

the maximum aspect ratio is suppressed and remains below $20 \%$.

So which transformation is superior? Over the target region $(\varphi<g(0))$, both transformations yield the same number of grid points. However, the two transformations produce different local effective resolutions. Figure $4 \mathrm{~b}$ illustrates the resolutions after the transformations. The solid and dotted lines represent the stretched degrees in latitudinal and longitudinal directions, respectively, from our proposed transformation. The longitudinal resolution is always less than the latitudinal resolution, so in our proposed transformation, the effective resolution is determined by the longitudinal resolution. In contrast, the broken line represents the grid interval from the Schmidt transformation, where the longitudinal and latitudinal resolutions at any local point are exactly the same because of isotropy.

From the perspective of truncating solution error, the maximum grid interval within the target region may govern physical performance. The maximum grid interval over the target region is represented by

$$
U=\sup _{-\pi / 2<\varphi<g(0)} \max \left(\frac{d \varphi}{d \phi}, \frac{\cos \varphi}{\cos \phi}\right) .
$$

For our proposed transformation, $U=\left.\frac{\cos \varphi}{\cos \phi}\right|_{\varphi=g(0)}$. For the Schmidt transformation, $U$ has a value identical to that in our proposed transformation as shown in 
(a)

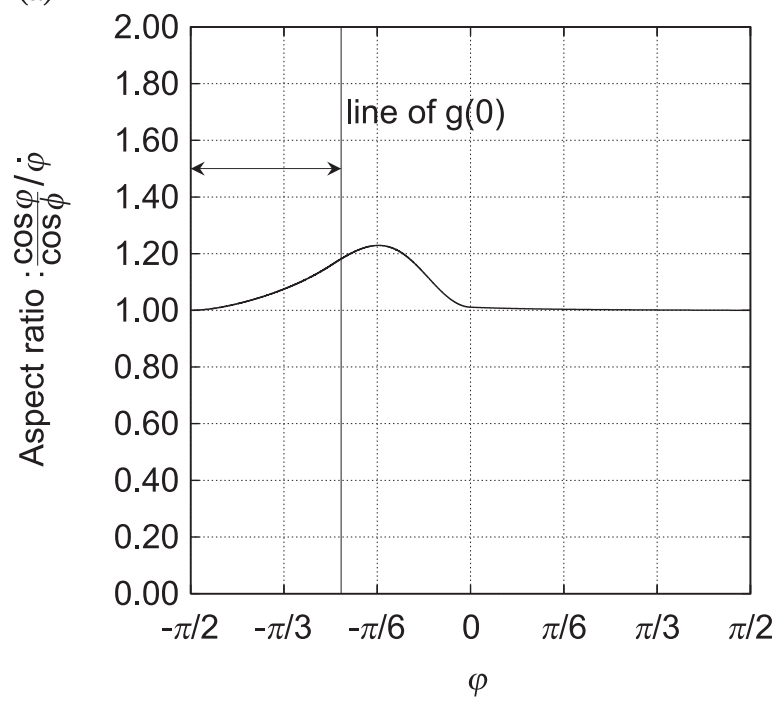

(b)

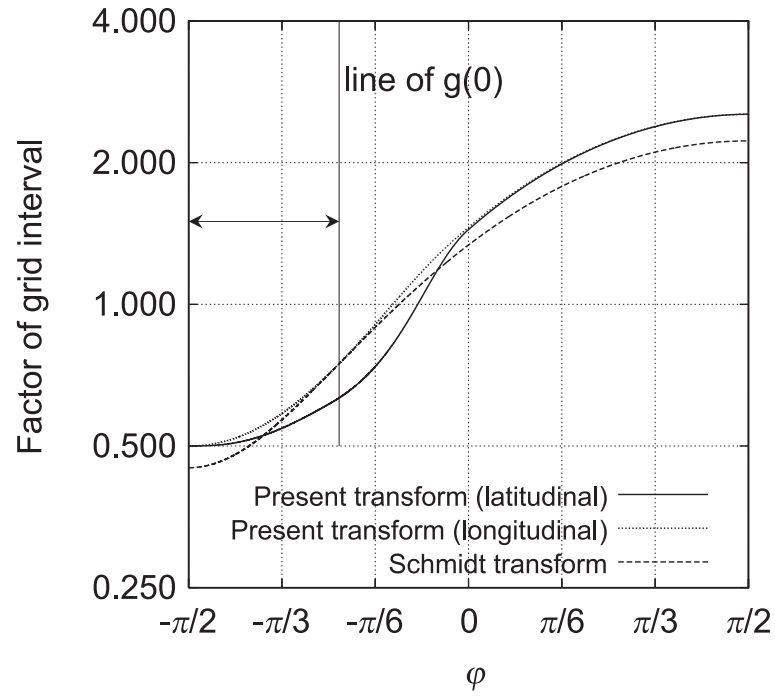

Fig. 4. (a) The aspect ration of grid element after the transformation $(\beta=1 / 2)$. (b) The local resolution after the transformation $(\beta=1 / 2)$.

Fig. 4b. This result suggests that the two transformation produce almost identical physical performances in the target region.

However, computational performance is determined by the minimum grid interval represented as

$$
L=\inf _{-\pi / 2<\phi<g(0)} \min \left(\frac{d \varphi}{d \phi}, \frac{\cos \varphi}{\cos \phi}\right) .
$$

(a)

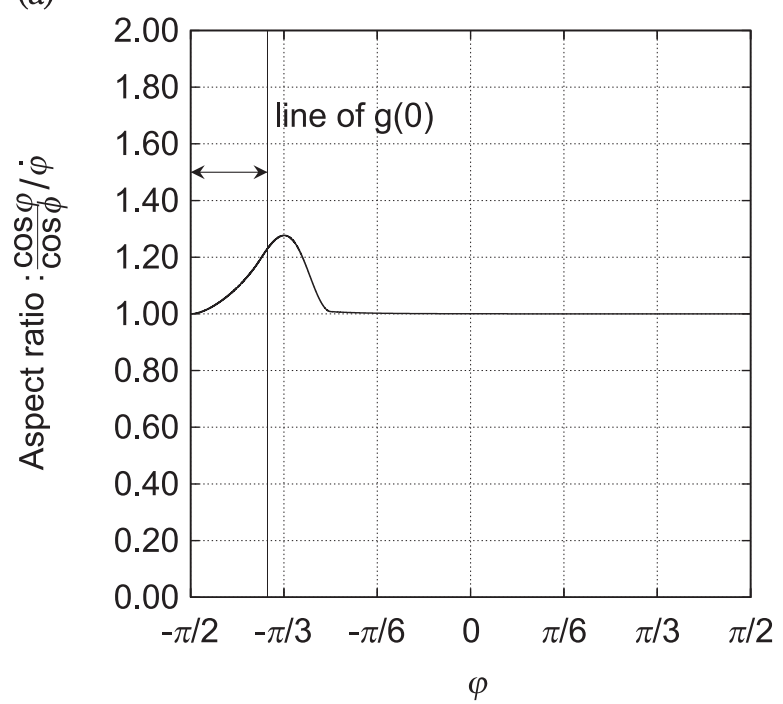

(b)

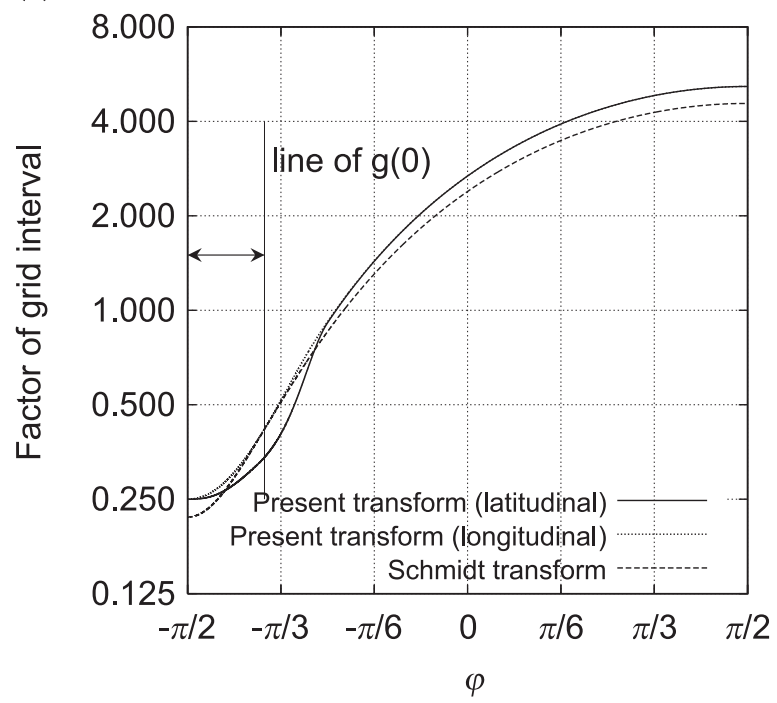

Fig. 5. Same figures as Fig. 4 but with $\beta=$ $1 / 4$.

From the perspective of the CFL condition, $L$ should be as large as possible. For our proposed transformation, $L=\beta(=1 / 2)$ at $\varphi=-\pi / 2$. For the Schmidt transformation, $L$ has a smaller value, as shown in Fig. 4b. In other words, the Schmidt transformation yields too fine a grid at $\varphi=-\pi / 2$ compared to our proposed transformation. Therefore, in terms of the CFL condition, our proposed transformation is superior to the Schmidt transformation. That is, the proposed transformation can use a larger time-step interval.

Figures 5 and 6 present aspect ratio and resolu- 
(a)

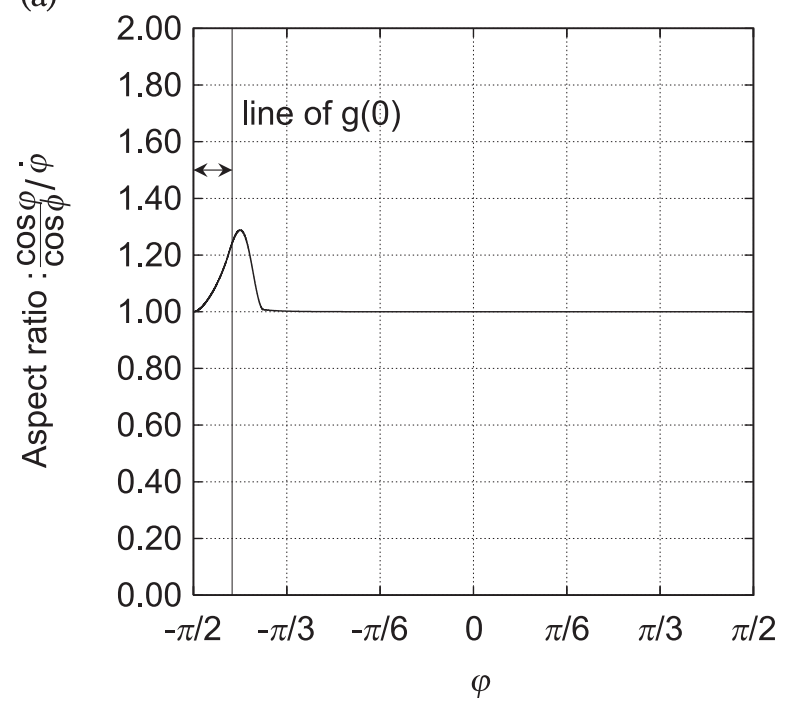

(b)

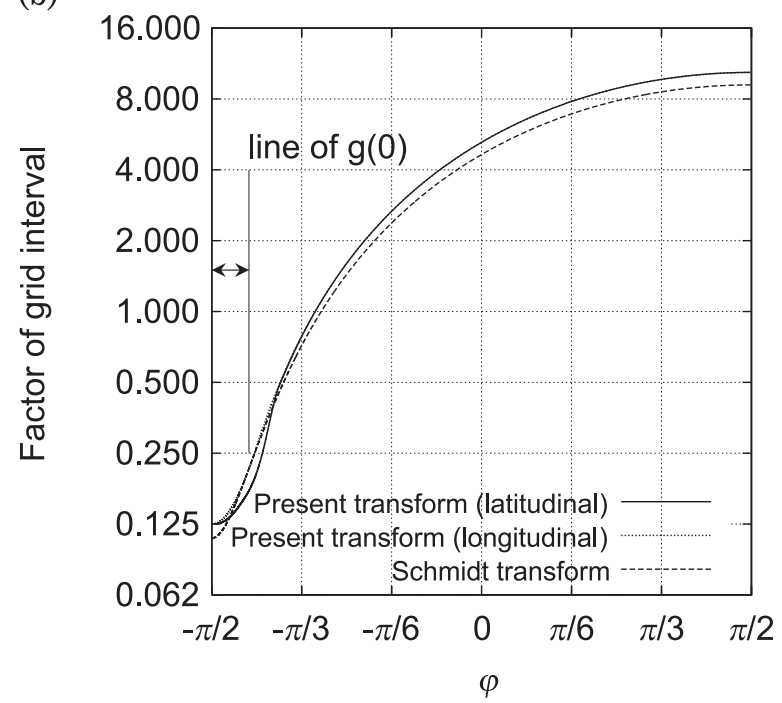

Fig. 6. Same figures as Fig. 4 but with $\beta=$ $1 / 8$.

tion with parameters $\beta=1 / 4$ and $1 / 8$, respectively. Even with a high degree of stretching, our proposed transformations produce a computational advantage compared to the Schmidt transformations.

We applied the transformation to an icosahedral grid. First, we modified the original icosahedral grid using spring dynamics (Tomita et al. 2002) to construct as homogeneous a grid as possible. After clustering the grid points near the South Pole using our proposed transformation with the parameter $\beta=1 / 4$, we rotated all the grid points so that the central point of the target region was located
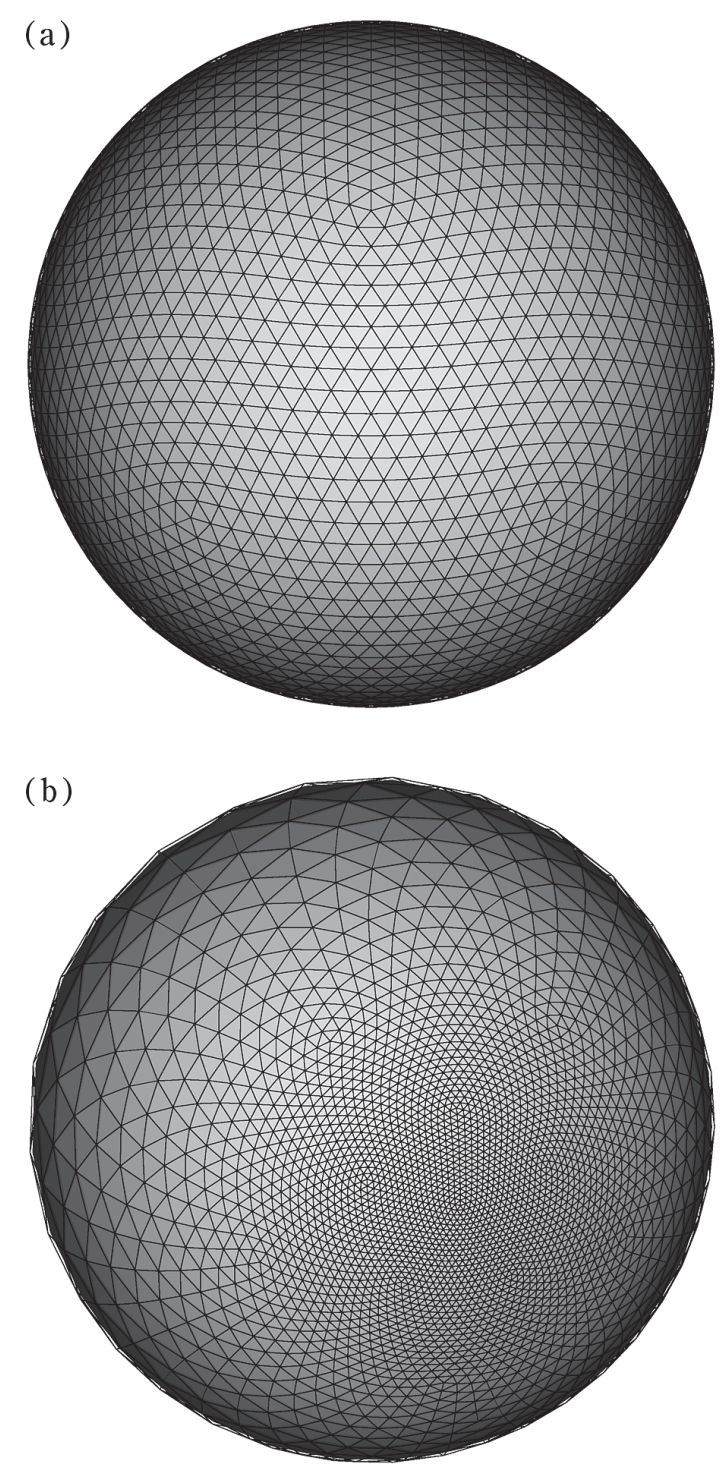

Fig. 7. (a) The icosahedral grid with four levels of grid division. (b) The stretched icosahedral grid by the present transformation.

at $(0 \mathrm{~N}, 0 \mathrm{E})$. Figures $7 \mathrm{a}$ and $\mathrm{b}$ show the icosahedral grid before the transformation and the stretched icosahedral grid after the transformation, respectively, with four levels of grid division. Note that the total grid number was calculated as $10 \cdot\left(2^{4}\right)^{2}$ $+2=2562$. The mean grid interval was approximately $500 \mathrm{~km}$, while that of the target region was approximately $120 \mathrm{~km}$. 


\subsection{The advection test}

This subsection discusses the advection tests we conducted according to Test Case 1 in Williamson et al. (1992). The purpose of this test was to evaluate the proposed transformation by investigating the error growth across the transition area and in the target region. We used an icosahedral grid with grid division level seven. We applied three cases: a uniform grid as a reference, a grid with stretched factor $\beta=1 / 2$, and a grid with stretched factor $\beta=1 / 4$. For the latter two grids, the centers of the target region were located at $(0 \mathrm{~N}, 90 \mathrm{E})$. We applied the upwind-biased scheme developed by Miura (2007) as the advection scheme on the ico- sahedral grid. We set the cosine bell distribution of the height field at $(0 \mathrm{~N}, 0 \mathrm{E})$ as the initial condition. This distribution was advected eastward, and the integration time was 12 days.

Figures $8 \mathrm{a}$ and $\mathrm{b}$ present the results with $\beta=$ $1 / 2$ and $\beta=1 / 4$, respectively. As shown in Fig. 8 , the cosine bell height field was smoothly advected across the transition zone without any noise in both cases. For the case of $\beta=1 / 2$, dissipation and dispersion errors barely appeared during the total integration time, while for the case of $\beta=1 / 4$, a slight dispersion error appeared in the latter half, e.g., at days 8 and 10. This was generated across the coarser grid hemisphere, where the grid inter-

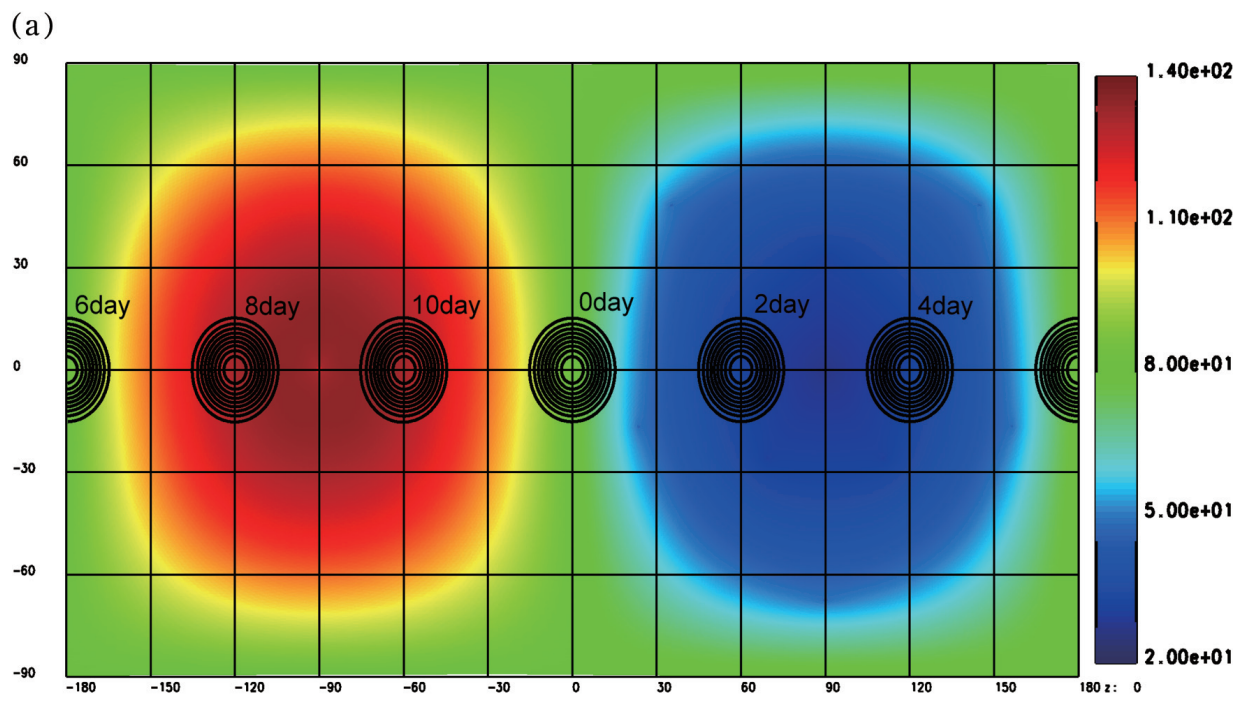

(b)

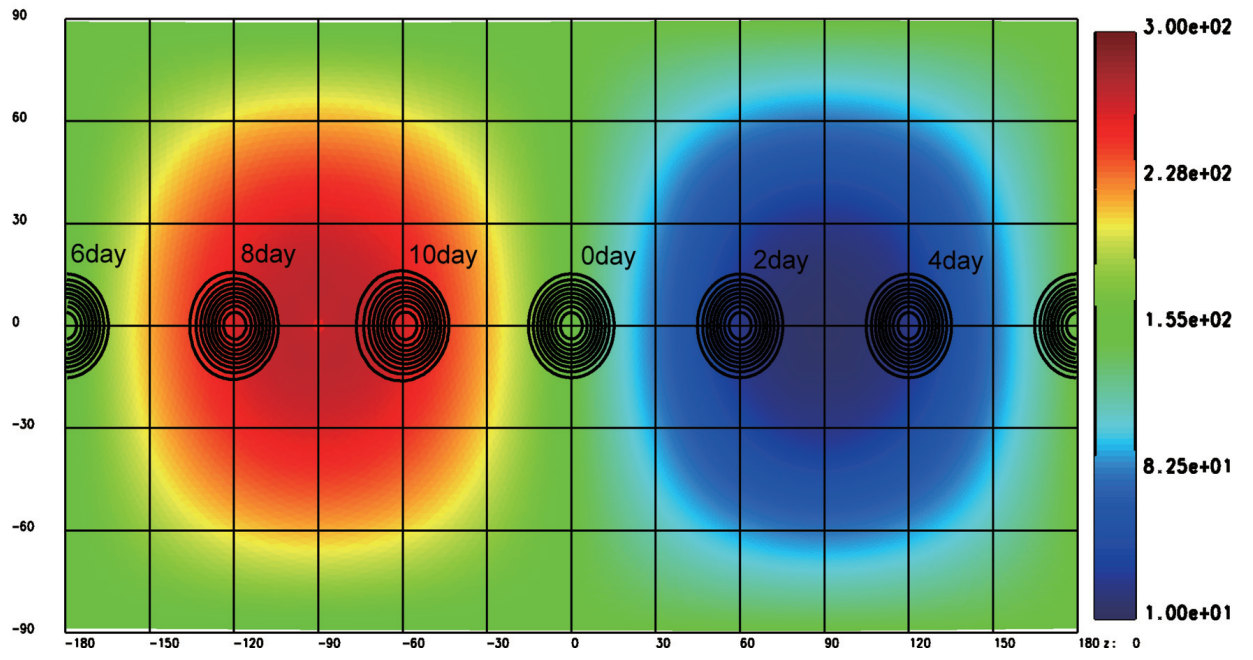

Fig. 8. The results of Test Case 1 in Williamson et al. (1992). Shading indicates local grid intervals. Contour lines indicate the height field for days $0,2,4,6,8$, and 10 . (a) $\beta=1 / 2$. (b) $\beta=1 / 4$. 
val varied from 150 to $300 \mathrm{~km}$.

Figure 9a gives the temporal history of $l_{2}$ norm error over the integration time for the three cases. Error grew gradually in the case of the uniform grid. In contrast, in the two stretched grids, error increased from the beginning to day 2 , the error level was maintained over the target region, and error increased again in the outer area. The error level of $\beta=1 / 4$ became higher than that of $\beta=1 / 2$ within the initial 2 days because the grid interval of $\beta=1 / 4$ was larger than that of $\beta=1 / 2$ in the transition area. However, as shown in Fig. 9b, the error growth rate $d l_{2} / d t$ of $\beta=1 / 4$ in the target region was less than that of $\beta=1 / 2$. Thus, the accuracy of $\beta=1 / 4$ was higher than that of $\beta=1 / 2$ in the target region. Note that the error growth rates of the stretched grids in the target region were lower than that of the uniform grid.

(a)

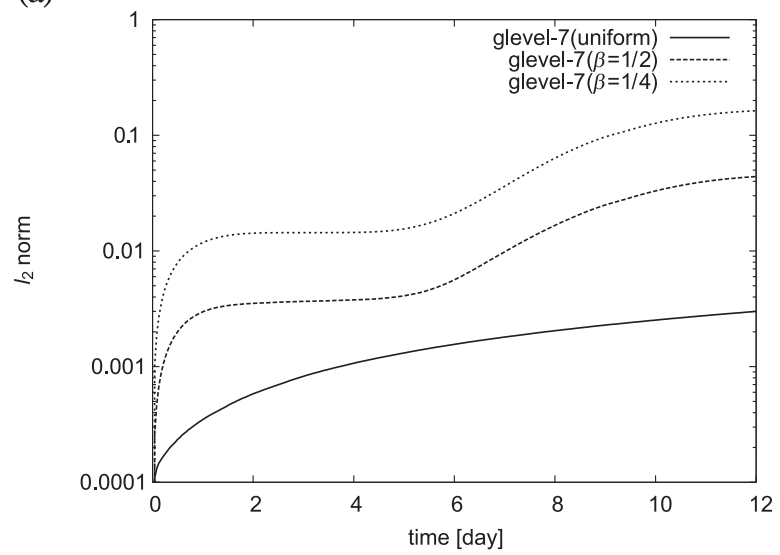

(b)

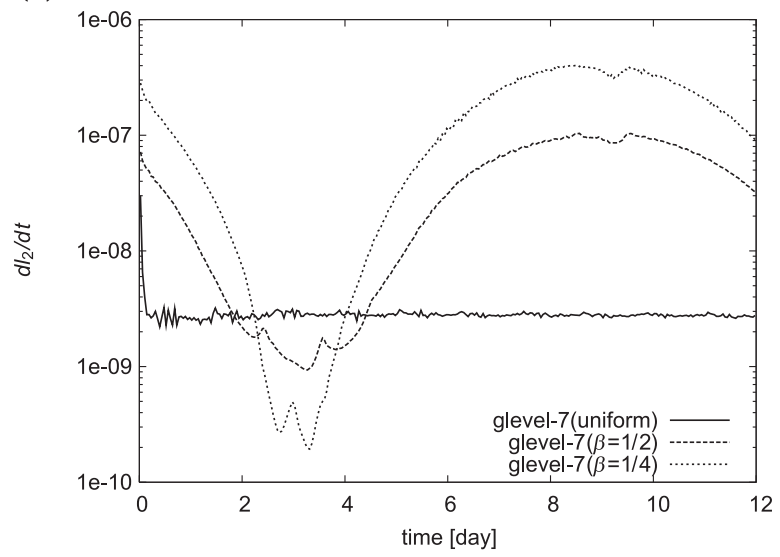

Fig. 9. (a) The time history of $l_{2}$ norm error. (b) The time history of $d l_{2} / d t$.

\section{Concluding remarks}

We proposed a new grid transformation on a sphere for the stretched grid system. We formulated the system as the solution to a variational problem, in which isotropy and homogeneity are compromised. As a result, transform functions are given by the solutions to a nonlinear second-order ordinary differential equation with two boundary conditions. We reformulated it as a set of first order equations with two initial conditions and numerically solved it. The Schmidt transformation is a special case of the present solutions when homogeneity is neglected.

Our proposed grid transformation yields a computational advantage over the Schmidt transformation. Both transformations produced the same total number of grid points in the target region, but while the Schmidt transformation minimized the grid interval too much, the proposed transformation reduced this problem.

We set out simple but reasonable weighting functions of isotropy $A$ and homogeneity $B$; the simple forms are given as Eqs. (34) and (35). Only one parameter $\beta$ stands for the stretched factor. The target region is also clarified using the relationship of Eq. (33). The aspect ratio of grid intervals in all of the cases were within $20 \%$, so the isotropy of grid element was maintained. Section 4 presented an application of the proposed transformation to the icosahedral grid. The new transformation can be applied to other homogeneous grid systems such as the cubic grid (e.g., non-conformal grids such as a gnomonic grid, Rancic et al. 1996, and the Kurihara reduced grid, Kurihara 1965).

Section 1 clarified how stretched grid systems generated from quasi-homogeneous grids are useful for local weather prediction and regional climate projection. One main advantage over the nesting method is that the same dynamical scheme could be used globally without any special modifications; that is, it makes the unified model described by (Côté et al. 1998a, b) possible. Additionally, as new models are being developed, it will allow many test runs to validate possible new physical schemes for implementation at minimal computational cost. The author has already applied the stretched grid in NICAM during the development stage of a new microphysical scheme, and the results will appear in a subsequent paper. 


\section{Acknowledgments}

All calculations in this study were performed on the Earth Simulator at the Earth Simulator Center. A portion of this work was supported by Core Research for Evolutional Science and Technology (CREST), Japan Science and Technology Agency.

\section{References}

Baumgardner, J.R. and P.O. Frederickson, 1985: Icosahedral discretization of the two-sphere. SIAM J. Numer. Anal., 22, 1107-1115.

Côté, J., G. Desmarais, S. Granvel, A. M'ethot, A. Patoine, M. Roch, and A. Staniforthk 1998: The operational CMC-MRB global environmental multiscale (GEM) model. Part II : Results. Mon. Wea. Rev., 126, 1397-1418.

Côté, J., S. Granvel, A. M'ethot, and A. Patoine, 1998b: The operational CMC-MRB global environmental multiscale (GEM) model. Part I: Design considerations and formulation. Mon. Wea. Rev., 126, 1373-1395.

Courtier, P. and J-F. Geleyn, 1988: A global numerical weather prediction model with variable resolution: Application to the shallow-water equations. Quart. J. Roy. Meteor. Soc., 114, 1321-1346.

Cullen, M.J.P. and C.D. Hall, 1979: Forecasting and general circulation results from finite element models. Quart. J. Roy. Meteor. Soc., 100, 571-592.

Heikes, R.H. and D.A. Randall, 1995a: Numerical integration of the shallow-water equations on a twisted icosahedral grid. Part I: basic design and results of tests. Mon. Wea. Rev., 123, 1862-1880.

Heikes, R.H. and D.A. Randall, 1995b: Numerical Integration of the Shallow-Water Equations on a Twisted Icosahedral Grid. Part II: A Detailed Description of the Grid and Analysis of Numerical Accuracy. Mon. Wea. Rev., 123, 1881-1887.

Iga, S., H. Tomita, M. Satoh, and K. Goto, 2007: Mountain-wave-like spurious waves due to inconsistency of horizontal and vertical resolution associated with cold fronts. Mon. Wea. Rev., 135, 2629-2641.

Kurihara, Y., 1965: Numerical integration of the primitive equations on a spherical grid. Mon. Wea. Rev., 93, 399-415.

Majewski, D., D. Liermann, P. Prohl, B. Ritter, M. Buchhold, T. Hanisch, G. Paul, and W. Wergen, 2002: The operational global icosahedral-hexagonal gridpoint model GME: Description and highresolution Tests. Mon. Wea. Rev., 130, 319-338.

Masuda, Y. and H. Ohnishi, 1986: An integration scheme of the primitive equation model with an icosahedral-hexagonal grid system and its application to the shallow water equations. In
Short- and Medium-Range Numerical Weather Prediction. Collection of Papers Presented at the WMO/IUGG NWP Symposium, Tokyo, Aug. 4-8, 317-326.

McGregor, J.L. 1997a: Preliminary climate simulations with a variable resolution global GCM. International Workshop on Regional Modeling of the General Monsoon System in Asia, 65-69.

McGregor, J.L. 1997b: Regional climate modelling. Meteor. Atmos. Phys., 63, 105-117.

McGregor, J.L., 1999: Regional modelling at CAR: recent development. Parallel computing in meteorology and oceanography: BMRC Research Report No.75, 43-48.

McGregor, J.L. and M.R. Dix, 1997: Development of a global conformal-cubic primitive eqations model. A. Staniforth, Ed., Research Activities in Atmospheric and Oceanic Modelling Report No.25, WGNE Report, 3.27-3.28.

McGregor, J.L. and M.R. Dix, 1998: A conformal-cubic atmospheric general circulation model. A. Staniforth, Ed. Research Activities in Atmospheric and Oceanic Modelling Report No.27, WGNE Report, 3.22-3.23.

McGregor, J.L. and J.J. Katzfey, 1998: NWP experiments with a variable-resolution conformal-cubic primitive model. A. Staniforth, Ed. Research Activities in Atmospheric and Oceanic Modelling Report No.27, WGNE Report, 3.20-3.21.

McGregor, J.L. and J.J. Katzfey, 1999: Simulation typhoon recurvature with a variable-resolution conformal-cubic model. A. Staniforth, Ed. Research Activities in Atmospheric and Oceanic Modelling Report No.28, WGNE Report, 3.19-3.20.

Miura, H., 2007: An upwind biased conservative scheme for spherical hexagonal-pentagonal grids. Mon. Wea. Rev., 135, 4038-4044.

Miura, H., M. Satoh, T. Nasuno, A.T. Noda, and K. Oouchi, 2007a: A madden-julian oscillation event realistically simulated by a global cloud-resolving model. Science, 318, 1763-1765.

Miura, H., M. Satoh, H. Tomita, T. Nasuno, S. Iga, and A. Noda, 2007: A short-duration global cloudresolving simulation with a realistic land sea distribution. Geophys. Res. Lett., 34, L02804, doi: 10.1029/2006GL027448.

Miura, H., H. Tomita, T. Nasuno, S. Iga, M. Satoh, and T. Matsuno, 2005: A climate sensitivity test using a global cloud resolving model under an aqua planet condition. Geophys. Res. Lett., 32, L19717, doi:10.1029/2005GL023672.

Nasuno, T., H. Tomita, S. Iga, H. Miura, and M. Satoh, 2007: Multiscale organization of convection simulated with explicit cloud processes on an aquaplanet. J. Atmos. Sci., 64, 1902-1921.

Rancic, M., R.J. Purser, and F. Mesinger, 1996: A global shallow-water model using an expanded spheri- 
cal cube: Gnomonic versus conformal coordinates. Quart. J. Roy. Meteor. Soc., 122, 959-982.

Ringler, T.D., R.H. Heikes, and D.A. Randall, 2000: Modeling the atmospheric general circulation using a spherical geodesic grid: A new class of dynamical cores. Mon. Wea. Rev., 128, 24712490.

Sadourny, R., A. Arakawa, and Y. Mintz, 1968: Integration of the nondivergent barotropic vorticity equation with an icosahedral hexagonal grid for the sphere. Mon. Wea. Rev., 96, 351-356.

Satoh, M., 2002: Conservative scheme for the compressible non-hydrostatic models with the horizontally explicit and vertically implicit time integration scheme. Mon. Wea. Rev., 130, 1227-1245.

Satoh, M., 2003: Conservative scheme for a compressible non-hydrostatic model with moist processes. Mon. Wea. Rev., 131, 1033-1050.

Satoh, M., T. Masuno, H. Tomita, H. Miura, T. Nasuno, and S. Iga, 2008: Nonhydrostatic icosahedaral atmospheric model (NICAM) for global cloud resolving simulations. J. Comput. Phys., the special issue of Predicting Weather, Climate and Extreme Events, 227, 3486-3514. doi:10.1016/j.jcp2007.02. 006.

Schmidt, F., 1977: Variable fine mesh in spectral global model. Beitr. Phys. Atmos., 50, 211-217.
Stuhne, G.R. and W.R. Peltier, 1996: Vortex erosion and amalgamation in a new model of large scale flow on the shere. J. Comput. Phys., 128, 58-81.

Tomita, H., H. Miura, S. Iga, T. Nasuno, and M. Satoh, 2005: A global cloud-resolving simulation: Preliminary results from an aqua planet experiment. Geophys. Res. Lett., 32, L08805, doi:10.1029/2005 GL022459.

Tomita, H. and M. Satoh, 2004: A new dynamical framework of nonhydrostatic global model using the icosahedral grid. Fluid Dyn. Res., 34, 357-400.

Tomita, H., M. Satoh, and K. Goto, 2002: An Optimization of the icosahedral grid modified by spring dynamics. J. Comput. Phys., 183, 307-331.

Tomita, H., M. Tsugawa, M. Satoh, and K. Goto, 2001: Shallow water model on a modified icosahedral geodesic grid by using spring dynamics. J. Comput. Phys., 174, 579-613.

Williamson, D.L., 1968: Integration of the barotropic vorticity equation on a spherical geodesic grid. Tellus, 20, 642-653.

Williamson, D.L., J.B. Drake, J.J. Hack, R. Jacob, and P.N. Swarztrauber, 1992: A standard test set for numerical approximations to the shallow water equations in spherical geometry. J. Comput. Phys., 102, 211-224. 